\title{
Effects of Smartphone-Delivered Positive-Word Stimulation on Depressed Mood in People with Subthreshold Depression: Protocol for a Pilot Randomized Controlled Trial
}

\author{
Hitomi Ejiri' \\ Hiroyuki Uchida ${ }^{2}$ \\ Kenji Tsuchiya ${ }^{1,3}$ \\ Kazuhiko Fujiwara ${ }^{4}$ \\ Senichiro Kikuchi ${ }^{1,3}$ \\ Kazuki Hirao (D) ${ }^{1,3}$
}

'School of Health Sciences, Faculty of Medicine, Gunma University, Maebashi, Japan; ${ }^{2}$ Department of Rehabilitation, Kurashiki Heisei Hospital, Kurashiki, Japan; ${ }^{3}$ Graduate School of Health Sciences, Gunma University, Maebashi, Japan; ${ }^{4}$ Department of Rehabilitation Sciences, Faculty of Rehabilitation Sciences, Nishikyusyu University, Kanzaki, Japan
Correspondence: Kazuki Hirao Graduate School of Health Sciences, Gunma University, 3-39-22 Showa, Maebashi, Gunma, 37I-85I4, Japan Tel/Fax +8I-27-220-8952

Email kazuki.hirao@gunma-u.ac.jp
Background: Major depressive disorder (MDD) is an important public health problem. Thus, preventive interventions against subthreshold depression (StD), which is one of the key risk factors for the development of MDD, are important. The study developed a smartphone application (SPSRS) that improves depressive symptoms in people with StD by automatically presenting positive word stimuli during videos. The SPSRS application has the potential to improve depressive symptoms in people with StDs. However, whether it can immediately improve depressed moods in people with StD is unclear. The study presents a protocol for investigating the immediate effects of the SPSRS application intervention on depressed mood in people with StD.

Methods: The study is designed as an open-label, parallel-group, and pilot randomized controlled trial (RCT). Thirty-two people who meet the criteria for StD (Beck Depression Inventory-II score of 10 points or more and fails to meet the diagnostic criteria for MDD) will be recruited and studied. It compares the immediate effects of the SPSRS application intervention (with positive word stimulus in a 10-minute video; $\mathrm{n}=16$ ) and YouTube application intervention (without positive word stimulus in 10 -minute video; $n=16$ ). The primary outcome is the change in the score for the Profile of Mood States 2nd Edition-Adult Short after 10 minutes of intervention. The secondary outcome is the change in the StateTrait Anxiety Inventory State after 10 min of intervention.

Discussion: The pilot RCT is the first to evaluate the immediate effects of the SPSRS application intervention on depressed mood in people with StD. The results are expected to provide a preliminary outcome of the immediate effect of the SPSRS application on the depressed mood of people with StD and aid in planning a full-scale trial.

Trial Registration: ClinicalTrials.gov; Identifier: NCT03864484.

Keywords: depression, subthreshold depression, depressed mood, mobile application, smartphone

\section{Introduction}

Major depressive disorder (MDD) is one of the most disease-burdened psychiatric disorders worldwide and is an important issue in public health. ${ }^{1,2}$ Therefore, many interventions have been proposed to treat MDD. ${ }^{3}$ However, even under optimal conditions, modern treatments are estimated to reduce only approximately one-third of MDD-related disease burdens. ${ }^{4}$ In addition, only approximately half of individuals treated with MDD fully recover, and these recovered individuals frequently 
eventually relapse. ${ }^{4}$ Another promising means of further reducing disease burdens related to MDD is prophylactic intervention that aims to prevent the development of MDD. ${ }^{5,6}$ Since the $1980 \mathrm{~s}$, many preventive programs have been developed toward this end. ${ }^{7}$ Many types of prevention have been developed, but those that target people with prodromes in particular can be an effective preventive strategy. ${ }^{3,6}$ Therefore, providing prophylactic intervention for subthreshold depression (StD), which is a prodrome of MDD, is important. StD fails to meet the diagnostic criteria for MDD but is characterized by clinically significant depressive symptoms. ${ }^{8}$ The prevalence of StD is $4.0 \%-53.2 \%,{ }^{9-12}$ which is an important risk factor for the development of MDD. ${ }^{13}$ Therefore, several effective intervention strategies for $\mathrm{StD}$ have been proposed. ${ }^{13,14}$ In particular, substantial evidence states that psychotherapy can reduce depressive symptoms in people with $\mathrm{StD}$ and prevent future developments of MDD. ${ }^{13,14}$ However, the practice of psychotherapy requires a qualified and specialized therapist, which limits access to treatment. ${ }^{15}$ In addition, patients may refuse the treatment they need because of the stigma, embarrassment, and prejudice associated with receiving psychotherapy. ${ }^{16,17}$

Interventions using smartphones may be a solution to these problems. Smartphone owners continue to increase worldwide, and many affordable or free applications for mental health are available. ${ }^{18,19}$ In addition, interventions through smartphone applications may reduce barriers to hospital visits and face-to-face treatment. ${ }^{18}$ As a result, the scheme has the potential to provide access to people who cannot be reached by other, traditional forms of treatment and to provide cost-effective interventions at the global scale. Several smartphone applications for depressive disorder have been developed due to these advantages. ${ }^{18,19}$ However, previous scholars suggested that people with StD require disease-specific interventions. ${ }^{20}$ Nonetheless, no smartphone application has been developed specifically for individuals with $\mathrm{StD}{ }^{18}$ Therefore, the researchers developed a smartphone application (SPSRS) dedicated to $\mathrm{StD}$, which aims to improve depressive symptoms among people with $\mathrm{StD}$, thus preventing the progression to MDD. ${ }^{21}$ The SPSRS application is a free video playback application designed with evidence backed by a word stimulus research. ${ }^{9,22-24}$ One of the biggest features is that an individual can receive a positive word stimulus while watching a video. In addition, the SPSRS application uses the YouTube Application Programming Interface. As a result, the SPSRS application can take advantage of videos uploaded to YouTube, which will automatically display a positive word stimulus. These benefits enable people with StD to select videos that suit their tastes and work toward reducing depressive symptoms. Encouragingly, preliminary evidence indicates that the SPSRS application can improve depressive symptoms in people with StD through a 5-week intervention. ${ }^{21,25}$ However, whether the SPSRS application can immediately improve one of the central symptoms of $\mathrm{StD}$, namely, depressed mood, is unclear. Many interventions focus on repetitive cumulative effects rather than immediate effects. However, the immediate effect of the intervention has important clinical and methodological significance. Cumulative effects may help address chronic depressive symptoms but unable to cope with the depressed mood that results from unpredictable stimuli that frequently occur in daily life. In addition, given psychological factors, such as conditioning and expectations, an immediate improvement in depressed mood may motivate people with StD to continue treatment and promote participation in long-term interventions that produce additional treatment effects. Therefore, the paper presents a pilot randomized controlled trial (RCT) of the immediate effects of SPSRS applications on depressed moods in people with StD.

\section{Materials and Methods}

\section{Trial Design}

The study is designed as an open-label, parallel-group, pilot RCT. The study compares the immediate effects of SPSRS application intervention (with positive word stimulus) and YouTube application intervention (without positive word stimulus). To prevent bias associated with the study design, this protocol followed the Standard Protocol Items: Recommendations for Interventional Trials (SPIRIT) 2013 statement. $^{26}$

Participants are selected according to the following eligibility criteria:

(A) Inclusion criteria.

1. Male and female

2. 20 to 39 years old

3. Beck Depression Inventory-II (BDI-II) score of 10 points or more ${ }^{27,28}$ 
4. Persons with written informed consent prior to participation.

(B) Exclusion criteria.

1. Persons who have been diagnosed with a psychiatric disorder at least once in their lifetime, regardless of the type of psychiatric disorder.

2. Currently receiving treatment from a professional for mental health issues.

3. Vision or hearing problems that interfere with daily life.

4. With a major depressive episode in the last two weeks according to the Mini-International Neuropsychiatric Interview. $^{29}$

We included people aged 20-39 years in this study because they are one of the population categories most likely to develop new MDD. A previous systematic review suggested that $\mathrm{StD}$ is widespread in all generations, ${ }^{30,31}$ but the 20-39 age group is most likely to develop new MDD. ${ }^{32,33}$ In fact, previous RCTs on StD have been conducted among this age group. ${ }^{34}$ For these reasons, we chose the 20-39 age group for our study.

\section{Interventions}

\section{Experimental Group}

The experimental group receives video-viewing intervention using the SPSRS application, which is a smartphone application. ${ }^{21}$ SPSRS is programmed to present $150 \mathrm{~ms}$ of positive words after presenting $17 \mathrm{~ms}$ of self-confidenceboosting words in videos.

These words are repeated every $5 \mathrm{~s}$. The words to increase self-confidence are "can," "let us try," "good luck," "able," and "do not worry". 9 These self-confidenceboosting words randomly appear in the four corners of the screen. The positive words are "nice," "great," "fantastic," "satisfactory," and "enjoyable". 22 These words are displayed at the center of the screen. Interveners use the SPSRS application according to the operating manual. The participants will use an iPhone managed by interveners to watch a pre-determined 10-minute video (basketball play video). Therefore, participants will be accompanied by the researchers when watching the video. The duration of video viewing in this study was determined from the results of a previous study (pilot RCT) that investigated the effects of the SPSRS application on people with StD. ${ }^{25}$

\section{Control Group}

The control group uses the same iPhone as the experimental group. The control group uses the YouTube application to receive video-viewing intervention. The control group videos do not show confidence-boosting and positive words. The control group watches the same video as the experimental group for $10 \mathrm{~min}$.

\section{Criteria for Discontinuing or Modifying Allocated Interventions}

If a participant meets any of the following conditions, then the research team will discontinue the intervention.

(1) If the participant wishes to discontinue the intervention.

(2) If the research team concludes that continuing the intervention is difficult due to deterioration in mental or physical health.

(3) If the entire clinical trial is discontinued.

(4) If the participant is ineligible after registration.

(5) If the research team concludes that the intervention is worthy of discontinuation for any reason.

The date and reason for discontinuation will be fully documented in a case report. The participants will not be considered to have dropped out of the study at the stage of discontinuing the intervention but will be requested to undergo primary and secondary outcome assessment. If a participant refuses to take the assessment or withdraws consent, then they are considered to have dropped out. The participants are excluded from intention-to-treat (ITT) analysis of the trial only if they are found unable to meet the eligibility criteria.

\section{Strategies for Monitoring and Improving Adherence to the Intervention Protocol}

To standardize the two interventions, the interveners will receive training in intervention using an intervention manual. In addition, to ensure the fidelity of the treatment, a periodical check will be conducted on the intervention methods for the experimental and control groups. Furthermore, the compliance rate for the intervention in the experimental and control groups will be evaluated using indirect and direct methods. ${ }^{35}$ As an indirect approach, the interveners complete a checklist of intervention procedures after 10 min of video viewing. The checklist is conducted to ensure that the interventions for the experimental or control group are conducted in a standardized manner and are included in the intervention manual. A researcher not involved in the intervention as a direct approach will conduct 
a checklist similar to the one completed by the interveners. The researcher discusses issues related to treatment delivery with interveners if deemed inconsistent.

\section{Relevant Concomitant Care and Interventions Permitted/ Prohibited During the Trial}

The study does not prohibit other forms of care from an ethical point of view.

\section{Outcomes}

The primary outcome is the change in the score for the Profile of Mood States 2nd Edition-Adult Short (POMS 2-A Short) after 10 min of intervention. ${ }^{36,37}$ POMS 2-A Short ask questions about current mood and allow change in the state of mood to be monitored. Therefore, we set POMS 2-A Short as the primary outcome to observe the immediate effect of the SPSRS application on mood state. The secondary outcome is the change in the State-Trait Anxiety Inventory State (STAIS) after 10 min of intervention. ${ }^{38-40}$

\section{Participant Timeline}

This study will be held at Gunma University in Gunma Prefecture, Japan. Potential participants will be evaluated for eligibility through interviews and questionnaires, and those eligible will be enrolled into the study after baseline evaluation (POMS 2-A Short and STAI-S). The baseline assessment will take place within one week after the eligibility assessment is conducted and would be conducted by the researchers. After eligibility and baseline assessments, participants will be randomly assigned to the experimental or control group by the central registration center at Kurashiki Heisei Hospital. Thereafter, the experimental and control groups will receive a 10-minute video viewing intervention. Participants will complete a second POMS 2-A Short and STAI-S immediately after the 10-minutes intervention. Figure 1 presents the trial period via a CONSORT diagram, ${ }^{41}$ whereas Table 1 provides the SPIRIT template. ${ }^{26}$

\section{Sample Size}

Data on the immediate effect of the SPSRS application on people with StD are lacking. Thus, estimating the sample size based on actual data is difficult. Although a pilot study does not require a formal sample size calculation, ${ }^{42}$ the number of samples in a pilot study with the objective of conducting a subsequent full-scale study should be 1520 individuals. ${ }^{43}$ Therefore, the study intends to recruit 16 participants per group for a total of 32 participants.

\section{Study Setting and Recruitment}

The trial is conducted at Gunma University in Gunma Prefecture, Japan from February 2021 to March 2023. The recruitment of potential participants is conducted on the basis of several recruitment strategies. Advertisement is widely implemented by posting advertisement flyers in

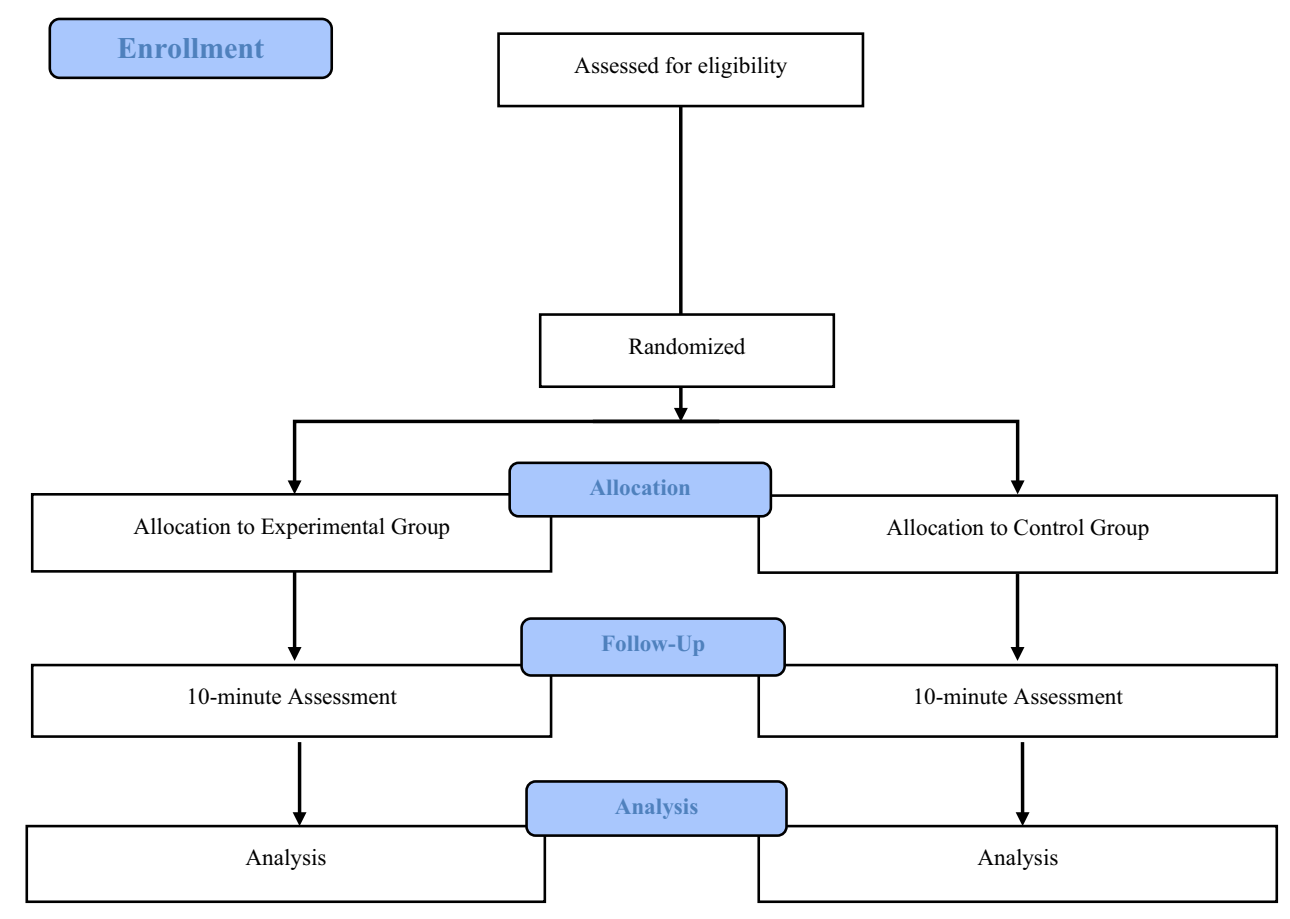

Figure I CONSORT flowchart of the study design. 
Table I Assessment Schedule

\begin{tabular}{|c|c|c|c|c|c|}
\hline \multirow[t]{3}{*}{ Timepoint } & \multicolumn{5}{|c|}{ Study Period } \\
\hline & \multirow{2}{*}{$\begin{array}{c}\text { Enrolment } \\
-t_{I}\end{array}$} & \multirow{2}{*}{$\begin{array}{c}\text { Baseline } \\
0\end{array}$} & \multirow{2}{*}{$\begin{array}{c}\text { Allocation } \\
0\end{array}$} & \multicolumn{2}{|c|}{ Intervention } \\
\hline & & & & $\frac{t_{l}}{\text { Before intervention }}$ & $\begin{array}{c}t_{2} \\
\text { After } 10 \mathrm{~min}\end{array}$ \\
\hline $\begin{array}{l}\text { Enrolment } \\
\text { Eligibility screen } \\
\text { Informed consent } \\
\text { Allocation }\end{array}$ & $\begin{array}{l}x \\
x\end{array}$ & & $x$ & & \\
\hline $\begin{array}{l}\text { Interventions } \\
\text { Experimental } \\
\text { group } \\
\text { Control group }\end{array}$ & & & & & \\
\hline $\begin{array}{l}\text { Assessments } \\
\text { Demographics } \\
\text { POMS 2-A Short } \\
\text { STAI-S }\end{array}$ & $x$ & $\begin{array}{l}x \\
x\end{array}$ & & & $\begin{array}{l}x \\
x\end{array}$ \\
\hline
\end{tabular}

Abbreviations: POMS 2-A Short, The Profile of Mood States 2nd Edition-Adult Short; STAI-S, The State-Trait Anxiety Inventory State.

the facilities of Gunma University. In addition, research participation is facilitated through email and social networking services. All materials contain a dedicated e-mail address for contacting the research team for further information about the study.

\section{Allocation}

Participants are randomly assigned to the experimental or control group in a 1:1 ratio after responding to the eligibility check and baseline assessment. A computer (Excel computer software) is used to generate a randomized list by the permuted block randomization method. The block size is not disclosed until the end of the trial to ensure concealment of the assignment. The generation of the randomized list is conducted by a third party not involved in the study. The randomized list is provided to a central registry center that is set up at Kurashiki Heisei Hospital for random assignment. Selection bias is thus avoided through these steps.

\section{Blinding}

Blinding participants and interveners is difficult because the interventions in the experimental and control groups are distinct. In addition, the small number of research staff renders the maintenance of rater blinding difficult. Therefore, the trial is planned as an open label trial. However, baseline assessment is performed before randomization. In addition, measure outcomes use standardized methods and implement procedures to audit and maintain data quality. Throughout these processes, to reducing the possibility of bias as much as possible is targeted.

\section{Data Collection}

To ensure accurate, complete, and reliable data, the following steps are conducted. (1) Training sessions on data collection and data storage are conducted. (2) Regular meetings are held to discuss issues related to the conduct of the study. (3) The telephone, e-mail, and communication media are used to enable consultation during the study with the principal investigator and co-investigators. The outcome assessment follows the schedule, as shown in Table 1 . The study collects demographic data and lifestyle characteristics, such as age, gender, sleep time, smoking habits, exercise habits, and drinking habits as part of the baseline assessment.

\section{Mini-International Neuropsychiatric Interview (MINI)}

The MINI is a structured diagnostic interview based on DSMIV and ICD-10. ${ }^{29}$ The Japanese version of MINI is designed with 16 psychiatric diagnostic modules. Respondents may respond with "yes" or "no" only. The reliability and validity of the MINI were reported elsewhere. ${ }^{29}$

\section{Beck Depression Inventory-II (BDI-II)}

The BDI-II is a 21 -item self-administered questionnaire that measures the severity of depressive symptoms. ${ }^{44}$ 
Items are rated using a 4-point Likert scale ranging from 0 to 3 points. The total score ranges from 0 to 63 points. High scores indicate more depressive symptoms. The reliability and validity of BDI-II have been reported elsewhere. $^{44-46}$

\section{Profile of Mood States 2nd Edition-Adult Short (POMS 2-A Short)}

The POMS 2-A Short is a 35-item self-administered questionnaire used to measure mood states. ${ }^{36,37}$ Items are rated using a 5-point Likert-type scale $(0=$ not at all, $1=$ a little, $2=$ moderately, $3=$ quite a lot, and $4=$ extremely). The POMS 2-A Short assesses seven domains, namely, angerhostility, confusion-bewilderment, depression-dejection, fatigue-inertia, tension-anxiety, vigor-activity, and friendliness. High scores indicate better vigor-activity and friendliness are better but more severe symptoms of the five other domains. In addition, the Total Mood Disturbance (TMD) score is calculated from anger-hostility, confusionbewilderment, depression-dejection, fatigue-inertia, tension-anxiety, and vigor-activity. TDM is calculated based on a standard value (mean of 50 and standard deviation of 10). The reliability and validity of POMS 2-A Short have already been reported. ${ }^{36,37}$

\section{State-Trait Anxiety Inventory (STAI)}

The STAI is a self-administered questionnaire consisting of two scales that each assesses state anxiety and trait anxiety. $^{38-40}$ Each scale consists of 20 items. Items for STAI-S are scored using a 4-point Likert-type scale $(1=$ not at all, $2=$ somewhat, $3=$ moderately so, and $4=$ very much so) with the total score ranging from 20 to 80 points. The higher the score, the greater the state anxiety. Meanwhile, items for STAI-T are scored using a 4-point Likert-type scale $(1=$ almost never, $2=$ sometimes, $3=$ often, and $4=$ almost always) with the total score ranging from 20 to 80 points. The higher the score, the greater the trait anxiety. The reliability and validity of the STAI have been reported elsewhere. ${ }^{38-40}$

\section{Plans to Promote Participant Retention and Complete Follow-Up}

The study will inform the participants on the details of the study schedule and importance of the study to prevent participant dropout and facilitate follow-up. In addition, all interventions and outcome assessments will be conducted free of charge, thus minimizing the outcome assessment and information to be collected. Participants who meet the criteria for refusal to continue the intervention or discontinuation will be invited to participate in the post-intervention assessment. These options will be emphasized and explained to participants during informed consent. If a participant is scheduled to drop-out, then the research team will discuss with the participant to identify and resolve any problems that the participant may have in maintaining the study.

\section{Data Management, Confidentiality, and Access to Data}

Data entry and statistical analysis will be conducted using computers that are not connected to the Internet at Gunma University. The observed data will be entered into an Excel spreadsheet on the date of observation. All data entered will be stored on a password-protected USB stick. Two independent researchers perform data entry separately to ensure accuracy. As a next step, the data entered by researchers other than the two researchers who performed the data entry will be checked for accuracy, presence of missing data, and data consistency. All data obtained will be stored securely in Gunma University. Paper materials related to the assessments and interventions collected will be kept in an evaluation file and an intervention file for each participant. These materials will be stored inside a locked shelf at Gunma University. The contents of the USB memory stick will be saved with the paper survey materials. The identifying information of the participants is excluded from the paper file, whereas other identifying information is recorded as participant number to protect privacy and maintain confidentiality. In addition, information about an individual's identity is not entered into the electronic database. The name or other personally identifying information, such as informed consent, is stored in a locked cabinet separate from the survey materials identified by participant number. The paper files will be kept for 10 years after the publication of the paper. Access to these files is restricted to the research staff involved in the study. The data manager and principal investigator will address data-related issues and complete the data set for statistical analysis. Currently, no plans are in place to allow public access to the dataset. However, only the approved researcher of the principal investigator can access the dataset for secondary analysis, metaanalysis, or other similar reasons.

\section{Statistical Methods}

Data analysis is conducted on the basis of ITT. The study uses linear mixed model (LMMs) with the restricted 
maximum likelihood estimate method for repeated measures analysis to examine the effect of the SPSRS application intervention on the primary and secondary outcomes and compare the two groups. ${ }^{47}$ LMM can address the flaws that occur in clinical trials and can use all available data to estimate results. ${ }^{47}$ Therefore, LMM yields estimates with less bias compared to traditional statistical analysis methods. For the LMMs, group assignment (experimental or control), time (pre- or post-intervention) , and interaction between group assignment and time at evaluation are considered fixed-effect factors, whereas participants are random-effects factors. The model uses a type-III test for fixed effects. Statistical significance is set to $\mathrm{p}<0.05$ for a two-tailed test. At this time, no subgroup analysis is planned. Data analyses use the latest version of SPSS. The effect size (Hedge's g) is reported between groups. ${ }^{48,49}$ Furthermore, the effect size between groups is reported as pre-, post-, and adjusted effect sizes, which is the post-effect size minus the pre-effect size. ${ }^{50}$

\section{Data Monitoring and Auditing}

The interventions provided by the app are not invasive, such that a formal data management board was deemed unnecessary because the risks are minimal. In addition, interim analysis and data audit on the impact of the intervention are not planned.

\section{Harm and Ancillary and Post-Trial Care}

Previous studies that examined the long-term effects of the SPSRS application on people with StD did not report adverse events (AEs). ${ }^{21,25}$ Therefore, the occurrence of AEs in the present study, which examines the immediate effects of SPSRS applications, is considered unlikely. However, using these apps may cause psychological distress depending on the participant's state of mind. Therefore, if unexpected AEs related to the intervention occur during or at the end of the study, the principal investigator will respond with appropriate actions to document the relationship between the participant and intervention in addition to the details of the AEs. AEs are monitored systematically based on the methods used in previous studies. ${ }^{51,52}$ All participants are encouraged to identify signs of poor mental or physical health at the time of study enrollment and required to report voluntarily. The appearance of such symptoms is considered an AE. Details of the AE are immediately reported to the Ethical Review Board for Medical Research Involving Human Subjects of Gunma University to confirm the direction of the study. In addition, any serious AEs after the study will be handled with appropriate measures. To this end, the participants will be able to contact the investigators for up to one month after the study.

\section{Research Ethics Approval, Protocol Amendments, and Consent or Assent}

Ethical Review Board for Medical Research Involving Human Subjects of Gunma University (approval number: HS2020-157) approved the study. This trial will be conducted in accordance with the Declaration of Helsinki. Changes in protocol, including those in the study objectives, study design, participant populations, sample size, study procedures, or significant administrative changes, may be made with the approval of the Ethical Review Board for Medical Research Involving Human Subjects of Gunma University. The principal investigator or coinvestigator is responsible for ensuring informed consent from each participant. Before commencing the study, the principal investigator or co-investigator will briefly explain to the participant, using the study information material, that participation is voluntary and can be discontinued at any time. Furthermore, the potential risks and benefits of participation is outlined. Informed consent should be given before the intervention, and participants should be convinced of its risks and benefits in writing.

\section{Dissemination Policy}

The research team is committed to the full disclosure of the study results regardless of outcome. Dissemination of the results is achieved at several national and international conferences and published in national and international peer-reviewed journals. The study is conducted and reported in accordance with the CONSORT statement. ${ }^{41}$

\section{Discussion}

Treatment of MDD is important for its prevention, which bears a significantly negative economic impact on society. ${ }^{1,2}$ In addition, prophylactic intervention in people with StD, a prodrome of MDD, may reduce the future incidence of MDD. ${ }^{3,6}$ Although many prophylactic intervention strategies are currently effective in improving the depressive symptoms of $\mathrm{StD},{ }^{13,14}$ certain problems emerge (eg, restricted access to treatment, stigma, and embarrassment of receiving psychotherapy). ${ }^{15-17}$ Therefore, interventions that can address these issues are required, and smartphone-based preventative interventions may be a more accessible option. ${ }^{18}$ Smartphone- 
based preventative interventions that are easily accessible due to many people with StD living in a community may offer additional benefits in improving the depressed mood of people with StD. Therefore, the researchers developed SPSRS, which is a smartphone application for improving depressive symptoms in people with StD. ${ }^{21}$ The study aims to investigate the immediate effects of SPSRS applications on depressed mood in people with StD. Previous studies have suggested that depressive symptoms in people with StD may be alleviated through a 5-week SPSRS application intervention. ${ }^{21,25}$ However, cumulative interventions may be unable to cope with momentary increases in depressed mood as a result of unpredictable stimuli that frequently occur in daily life. This situation suggests the need for developing effective prevention methods against momentary increases in depressed mood. ${ }^{53}$ In addition, given certain psychological factors, such as conditioning and expectations, immediate improvement in depressed mood may motivate people with StD to continue treatment. Based on these perspectives, the immediate effects of the SPSRS application on people with StD point to very important clinical implications. The findings that will be obtained from the study will provide a tentative estimate of the immediate effectiveness of the SPSRS application intervention on the depressed mood of people with StD. Once confirmed, the SPSRS application intervention will be an important step in strongly justifying the use of the intervention on people with StD as a means for preventing the development of MDD.

One of the problems with the SPSRS application intervention is that the underlying mechanism of the improvement of depressive symptoms by SPSRS applications remains unclear. Thus, understanding the immediate effects of SPSRS applications on the depressed mood of people with StD can aid in the development and understanding of definitive inferences about the therapeutic mechanisms of SPSRS applications. Factors that disrupt the internal validity of the results, such as spontaneous remission from the disease (ie, from studies examining the immediate effects of an intervention) and regression to the mean (in clinical studies), are expected to be less those for studies designed to examine cumulative intervention effects. Therefore, understanding the immediate effects of SPSRS applications on the depressed mood of people with StD may provide a definitive reasoning and a unique opportunity for understanding the underlying mechanisms of StD.
The findings obtained from the study provide important pilot data for calculating the sample size for a full-scale trial. The objective of the pre-sample size calculation is mainly to determine the number of participants required to detect clinically relevant therapeutic effects. ${ }^{54}$ In this manner, research can be conducted with minimal resources without compromising scientific integrity and rigor. In addition, large-scale trials that expose an extreme number of people to new therapies and under-detectable trials, which may not achieve significant results, should be avoided. ${ }^{54}$ Therefore, the importance of sample sizing is widely asserted, and such calculations should be reported and justified in published papers. ${ }^{54}$ Preliminary research is frequently conducted to generate data for sample size calculations. This method is a particularly effective in the absence of data from previous studies. ${ }^{42}$

The study comes with certain limitations. First, it only observes the immediate intervention effects of the SPSRS application on the depressed mood of people with StD. Therefore, further research is required to determine the long-term effects of this application intervention. Second, the trial does not perform a formal sample size calculation due to the lack of data on the immediate effects of SPSRS application intervention on depressed mood in people with StD. This limitation may reduce the detection power of the pilot RCT. Thus, the results should be interpreted with consideration of the limits. However, calculating the sample size of future large-scale trials is possible based on the results of the pilot trial. Finally, the interveners, participants, and assessors have not been blinded, which can lead to biases. Given the nature of the intervention, blinding the interveners and participants is difficult. In a full-scale trial, however, the assessor should be blinded.

\section{Conclusions}

The pilot RCT is the first to investigate the immediate effects of the SPSRS application intervention on depressed mood in people with StD. If the results are positive, then the SPSRS application intervention can reduce depressed mood in people with $\mathrm{StD}$ and offer new intervention strategies to prevent the development of future MDDs. In addition, the pilot RCT is well-designed based on standardized and robust procedures. As such, it can provide the data required to plan a full-scale trial to test the immediate effects of the SPSRS application intervention on the depressed mood of people with StD. 


\section{Author Contributions}

All authors contributed to data analysis, drafting or revising the article, gave final approval of the version to be published, agreed to the submitted journal, and agree to be accountable for all aspects of the work.

\section{Funding}

This work was supported by JSPS KAKENHI Grant Number 19K19724.

\section{Disclosure}

The authors report no conflicts of interest in this work.

\section{References}

1. Gustavsson A, Svensson M, Jacobi F, et al. Cost of disorders of the brain in Europe 2010. Eur Neuropsychopharmacol. 2011;21 (10):718-779.

2. Mathers CD, Loncar D. Projections of global mortality and burden of disease from 2002 to 2030. PLoS Med. 2006;3(11):e442.

3. Otte C, Gold SM, Penninx BW, et al. Major depressive disorder. Nat Rev Dis Primers. 2016;2:16065.

4. Chisholm D, Sanderson K, Ayuso-Mateos JL, Saxena S. Reducing the global burden of depression: population-level analysis of intervention cost-effectiveness in 14 world regions. $B r J$ Psychiatry. 2004;184:393-403.

5. Bellón J, Moreno-Peral P, Motrico E, et al. Effectiveness of psychological and/or educational interventions to prevent the onset of episodes of depression: a systematic review of systematic reviews and meta-analyses. Prev Med. 2015;76(Suppl):S22-32.

6. van Zoonen K, Buntrock C, Ebert DD, et al. Preventing the onset of major depressive disorder: a meta-analytic review of psychological interventions. Int J Epidemiol. 2014;43(2):318-329.

7. Institute of Medicine Committee on Prevention of Mental Disorders. In: Mrazek PJ, Haggerty RJ, editors. Reducing Risks for Mental Disorders: Frontiers for Preventive Intervention Research. Washington (DC): National Academies Press (US); 1994.

8. Rodríguez MR, Nuevo R, Chatterji S, Ayuso-Mateos JL. Definitions and factors associated with subthreshold depressive conditions: a systematic review. BMC Psychiatry. 2012;12:181.

9. Takahashi K, Takada K, Inoue A, et al. Identification of common words to improve self-confidence in Japanese students with subthreshold depression. Int J Adolesc Med Health. 2019;31(3):1-7.

10. Vaccaro R, Borrelli P, Abbondanza S, et al. Subthreshold depression and clinically significant depression in an Italian population of 70 74-year-olds: prevalence and association with perceptions of self. Biomed Res Int. 2017;2017:3592359.

11. Meeks TW, Vahia IV, Lavretsky H, Kulkarni G, Jeste DV. A tune in "a minor" can "b major": a review of epidemiology, illness course, and public health implications of subthreshold depression in older adults. J Affect Disord. 2011;129(1-3):126-142.

12. Xiang X, Leggett A, Himle JA, Kales HC. Major depression and subthreshold depression among older adults receiving home care. $\mathrm{Am}$ J Geriatric Psychiatry. 2018;26(9):939-949.

13. Cuijpers P, Smit F, van Straten A. Psychological treatments of subthreshold depression: a meta-analytic review. Acta Psychiatr Scand. 2007;115(6):434-441.

14. Cuijpers P, Koole SL, van Dijke A, Roca M, Li J, Reynolds CF 3rd. Psychotherapy for subclinical depression: meta-analysis. $\mathrm{Br}$ J Psychiatry. 2014;205(4):268-274.
15. Zhou T, Li X, Pei Y, Gao J, Kong J. Internet-based cognitive behavioural therapy for subthreshold depression: a systematic review and meta-analysis. BMC Psychiatry. 2016;16(1):356.

16. Pedersen ER, Paves AP. Comparing perceived public stigma and personal stigma of mental health treatment seeking in a young adult sample. Psychiatry Res. 2014;219(1):143-150.

17. Cuijpers $P$, van Straten A, Andersson G. Internet-administered cognitive behavior therapy for health problems: a systematic review. J Behav Med. 2008;31(2):169-177.

18. Miralles I, Granell C, Díaz-Sanahuja L, et al. Smartphone apps for the treatment of mental disorders: systematic review. JMIR mHealth uHealth. 2020;8(4):e14897.

19. Grist R, Porter J, Stallard P. Mental health mobile apps for preadolescents and adolescents: a systematic review. J Med Internet Res. 2017;19(5):e176.

20. Takagaki K, Okamoto Y, Jinnin R, et al. Behavioral characteristics of subthreshold depression. J Affect Disord. 2014;168:472-475.

21. Takahashi K, Takada K, Hirao K. Feasibility and preliminary efficacy of a smartphone application intervention for subthreshold depression. Early Interv Psychiatry. 2019;13(1):133-136.

22. Takarada Y, Nozaki D. Maximal voluntary force strengthened by the enhancement of motor system state through barely visible priming words with reward. PLoS One. 2014;9(10):e109422.

23. Aoyama Y, Uchida H, Sugi Y, et al. Immediate effect of subliminal priming with positive reward stimuli on standing balance in healthy individuals: a randomized controlled trial. Medicine. 2017;96(28): e7494.

24. Aarts H, Custers R, Marien H. Preparing and motivating behavior outside of awareness. Science (New York, NY). 2008;319(5870):1639.

25. Kageyama K, Kato Y, Mesaki T, et al. Effects of video viewing smartphone application intervention involving positive word stimulation in people with subthreshold depression: a pilot randomized controlled trial. $J$ Affect Disord. 2020;282:74-81.

26. Chan AW, Tetzlaff JM, Gøtzsche PC, et al. SPIRIT 2013 explanation and elaboration: guidance for protocols of clinical trials. $B M J$ (Clinical Research Ed). 2013;346:e7586.

27. Takagaki K, Okamoto Y, Jinnin R, et al. Behavioral activation for late adolescents with subthreshold depression: a randomized controlled trial. Eur Child Adolesc Psychiatry. 2016;25(11):1171-1182.

28. Furukawa TA, Horikoshi M, Kawakami N, et al. Telephone cognitive-behavioral therapy for subthreshold depression and presenteeism in workplace: a randomized controlled trial. PLoS One. 2012;7(4):e35330.

29. Otsubo T, Tanaka K, Koda R, et al. Reliability and validity of Japanese version of the mini-international neuropsychiatric interview. Psychiatry Clin Neurosci. 2005;59(5):517-526.

30. Wesselhoeft R, Sørensen MJ, Heiervang ER, Bilenberg N. Subthreshold depression in children and adolescents - a systematic review. J Affect Disord. 2013;151(1):7-22.

31. Lee YY, Stockings EA, Harris MG, et al. The risk of developing major depression among individuals with subthreshold depression: a systematic review and meta-analysis of longitudinal cohort studies. Psychol Med. 2019;49(1):92-102.

32. Yu B, Zhang X, Wang C, Sun M, Jin L, Liu X. Trends in depression among adults in the United States, NHANES 2005-2016. J Affect Disord. 2020;263:609-620.

33. Julian LJ, Tonner C, Yelin E, et al. Cardiovascular and disease-related predictors of depression in systemic lupus erythematosus. Arthritis Care Res (Hoboken). 2011;63(4):542-549.

34. Yamamoto A, Tsujimoto E, Taketani R, Tsujii N, Shirakawa O, Ono $\mathrm{H}$. The effect of interpersonal counseling for subthreshold depression in undergraduates: an exploratory randomized controlled trial. Depress Res Treat. 2018;2018:4201897.

35. Perepletchikova F, Treat TA, Kazdin AE. Treatment integrity in psychotherapy research: analysis of the studies and examination of the associated factors. J Consult Clin Psychol. 2007;75(6):829-841. 
36. Yokoyama K, Watanabe K. Japanese Translation of POMS 2: Profile of Mood States. 2nd ed. Tokyo: Kaneko Shobo; 2015.

37. Heuchert J, McNair D. POMS 2. J North Tonawanda, NY: MultiHealth Systems Incorporated; 2012.

38. Spielberger C. State-Trait Anxiety Inventory for Adults. Sampler Set, Manual Set, Scoring Key. Palo Alto CA: Consulting Psychologists Press; 1983.

39. Spielberger C, Gorsuch R, Lushene R. STAI Manual for the StateTrait Anxiety Inventory. Palo Alto, CA: Consulting Psychologist Press; 1970.

40. Hidano T, Fukuhara M, Iwawaki S, Soga S, Spielberger C. State-Trait Anxiety Inventory-Form JYZ. Tokyo: JITSUMUKYOIKU-SHUPPAN Co. Ltd; 2000.

41. Moher D, Hopewell S, Schulz KF, et al. CONSORT 2010 explanation and elaboration: updated guidelines for reporting parallel group randomised trials. BMJ (Clinical Research Ed). 2010;340:c869.

42. Thabane L, Ma J, Chu R, et al. A tutorial on pilot studies: the what, why and how. BMC Med Res Methodol. 2010;10:1.

43. Hertzog MA. Considerations in determining sample size for pilot studies. Res Nurs Health. 2008;31(2):180-191.

44. Kojima M, Furukawa TA, Takahashi H, Kawai M, Nagaya T, Tokudome S. Cross-cultural validation of the Beck Depression Inventory-II in Japan. Psychiatry Res. 2002;110(3):291-299.

45. Kjaergaard M, Arfwedson wang CE, Waterloo K, Jorde R. A study of the psychometric properties of the Beck Depression Inventory-II, the Montgomery and Åsberg Depression Rating Scale, and the Hospital Anxiety and Depression Scale in a sample from a healthy population. Scand J Psychol. 2014;55(1):83-89.
46. Hiroe T, Kojima M, Yamamoto I, et al. Gradations of clinical severity and sensitivity to change assessed with the Beck Depression Inventory-II in Japanese patients with depression. Psychiatry Res. 2005;135(3):229-235.

47. Gueorguieva R, Krystal JH. Move over ANOVA: progress in analyzing repeated-measures data and its reflection in papers published in the archives of general psychiatry. Arch Gen Psychiatry. 2004;61(3):310-317.

48. Hedges LV. Distribution theory for glass's estimator of effect size and related estimators. J Educ Statistics. 1981;6(2):107-128.

49. Hedges LV, Olkin I. Statistical Methods for Meta-Analysis. New York: Academic Press; 1985.

50. Durlak JA. How to select, calculate, and interpret effect sizes. J Pediatr Psychol. 2009;34(9):917-928.

51. Kato Y, Kageyama K, Mesaki T, et al. Study protocol for a pilot randomized controlled trial on a smartphone application-based intervention for subthreshold depression: study protocol clinical trial (SPIRIT compliant). Medicine. 2020;99(4):e18934.

52. Galante J, Dufour G, Benton A, et al. Protocol for the Mindful Student Study: a randomised controlled trial of the provision of a mindfulness intervention to support university students' wellbeing and resilience to stress. BMJ Open. 2016;6(11):e012300.

53. Pemberton R, Fuller Tyszkiewicz MD. Factors contributing to depressive mood states in everyday life: a systematic review. $J$ Affect Disord. 2016;200:103-110.

54. Charles P, Giraudeau B, Dechartres A, Baron G, Ravaud P. Reporting of sample size calculation in randomised controlled trials: review. BMJ. 2009;338:b1732.
Neuropsychiatric Disease and Treatment

\section{Publish your work in this journal}

Neuropsychiatric Disease and Treatment is an international, peerreviewed journal of clinical therapeutics and pharmacology focusing on concise rapid reporting of clinical or pre-clinical studies on a range of neuropsychiatric and neurological disorders. This journal is indexed on PubMed Central, the 'PsycINFO' database and CAS, and

\section{Dovepress}

is the official journal of The International Neuropsychiatric Association (INA). The manuscript management system is completely online and includes a very quick and fair peer-review system, which is all easy to use. Visit http://www.dovepress.com/testimonials.php to read real quotes from published authors. 